\title{
Low-Cost Fabrication of a PDMS Microchannel Using an Improved Print-and-Peel (PAP) Method
}

\author{
Kristian July R. Yap, John Paul Niño A. Sanglay, Jan Samuel C. Matuba, and Rodgee F. Abaya
}

\begin{abstract}
Microfluidics involves fluid dynamics, controlled fluid manipulations, and design of devices or systems in microchannels with typical dimensions of 10 to 200 micrometers. The conventional fabrication method of polydimethylsiloxane (PDMS) microchannels is soft lithography, which involves an expensive process for the preparation of master. The study aims to fabricate microchannels using print-and-peel (PAP) method for simple and low-cost construction of microfluidic systems. Based on the PAP method, a PDMS microchannel is developed using a master based on inkjet ink relief printed on paper. The study improves on the existing methodologies for PAP method by devising a technique called "printing-over", which is reprinting of the same pattern over itself at a high precision by modifying the printing process. This can produce irregularly-shaped molds with dimensions that can reach up to 200 micrometer width and $\mathbf{1 4 . 8}$ micrometer height, with aspect ratio equal to 0.07 . Moreover, the aspect ratio is found to increase proportionally with number of printing-over runs. The microchannel produced from the mold has dimensions of 155 by 10 micrometers with aspect ratio of 0.06 . With a rough cost analysis, the capital and variable costs of the PAP inkjet method are significantly lower than that of photolithography.
\end{abstract}

Index Terms - Low-cost microfluidics, polydimethylsiloxane, print-and-peel method, inkjet printing.

\section{INTRODUCTION}

Microfluidics is the science that deals with the flow of liquids inside micrometer-size channels. This technology has found applications in various areas including biomedical engineering, cell biology, drug screening, chemical reaction engineering, and electrochemistry. While the main advantage of microfluidics is the reduction of costs by reducing fluid volumes, the conventional fabrication process requires specialized equipment in laboratories; and the material used, usually silicon chips, can be expensive to produce. Inducing controlled fluid flows in microfluidic channels is done using special equipment called microsyringe pumps, but these are also too expensive and are unavailable locally. The high costs associated with the fabrication of microfluidics consequently limits accessibility and thus, hinders its development especially in low-resource settings.

The conventional fabrication method of polydimethylsiloxane (PDMS) microchannels is soft lithography, which involves an expensive process for the

Manuscript received October 8, 2016; revised January 18, 2017. This work was supported in part by the UP Engineering Research and Development Foundation, Inc. (ERDFI) and the College of Engineering, University of the Philippines Diliman.

The authors are with University of the Philippines Diliman, Quezon City 1101, Philippines (e-mail: kristian.yap@gmail.com). preparation of master called photolithography. Photolithography is a patterning process where light is used to transfer a pattern from a mask to a photosensitive polymer layer, and this resulting pattern can either be etched into the underlying surface or used to define the patterning of a layer deposited onto the masked surface [1]. This process has been used for several years in fabrication of inorganic electronics; however, this process needs equipment and tools that are expensive and requires extremely clean conditions during manufacturing. This traditional process is suited for mass production, but it can be difficult and expensive for prototyping.

Low-cost microfluidics is an initiative to remove the technical and economic barriers to the technology microfluidics offers. By simplifying the fabrication process and using alternative materials that are simpler and easier to procure, low-cost microfluidics would also enable rapid prototyping in order to accelerate the developments made in the field. The print-and-peel (PAP) method, which uses a printer for the preparation of master, is among the methods being explored for a low-cost alternative method of fabrication. Because of direct printing of the masters from the computer-aided design generated patterns, PAP involves less steps compared to photolithography in the fabrication of mold. PDMS does not adhere to the materials composing the PAP-fabricated masters, thus, eliminating the need for perfluoroalkylation step usually done in photolithography to suppress permanent adhesion of the mold with the PDMS [2]. The limitations of PAP fabrication is set by the resolution of the printer and properties of the printing materials and substrate [3]. References [4]-[6] used laserjet printers while reference [7] used solid-wax printer for producing microchannels. On the other hand, inkjet printing is still largely unexplored despite its lower cost among different types of printers, having a disadvantage of producing relief features with low height. Modifications must be done on the ink or the substrate to obtain higher relief features. Reference [8] fabricated polymer hydrophobic barriers through inkjet printing. The dimensions of the hydrophobic barriers were 120 micrometers wide and 10 micrometers deep. Reference [9] demonstrated a patterning technique, through the creation of hole structures on insulator-coated substrates via inkjet printing, with height reaching only submicrometer and width of about 120 micrometers.

The objective of the study is to fabricate a simple, low-cost PDMS microchannel. Based on the PAP method, a PDMS microchannel is developed using a mold based on inkjet ink relief printed on paper. The research improves on the existing methodologies for PAP method by increasing the achievable aspect ratio of the microchannel. The inkjet printing process 
is modified such that reprinting of the same pattern is done without the need for refeeding of paper, which is also called printing-over. The effect of the printing-over on the width and aspect ratio of the mold is also determined. In order to induce a controlled pressure flow across the microchannel, a microsyringe pump prototype is developed in $\mathrm{LEGO}{ }^{\circledR}$.

\section{Methodology}

\section{A. Construction of Microsyringe Pump}

A microsyringe pump was prototyped using a LEGO® Mindstorms ${ }^{\circledR}$ NXT robotics kit and a $0.30-\mathrm{mL}$ insulin syringe $\left(30 \mathrm{steps} / \mathrm{cm}^{3}\right)$ with a 31 -gauge needle. Using LabVIEW, the LEGO® Mindstorms ${ }^{\circledR}$ NXT was programmed to control the motor to rotate with a minimum angular speed of $27 \mathrm{rad} / \mathrm{s}$.

\section{B. Inkjet Printing of Mold}

A commercial office inkjet printer (Canon IP 2870) with black pigment-based ink was used to print on a glossy-type sticker paper. The paper feed sensor was configured to be able to reprint repeatedly over the same pattern without refeeding the paper. A drafting software (Autodesk AutoCAD®) was used to design a microchannel pattern of straight lines with a minimum preset line weight. The printed patterns were air-dried for at least one (1) day.

\section{Mold Characterization and Selection}

Images of all printed patterns were taken using a light microscope (Euromex Novex B Series) with 50x magnification, and their widths were measured using a graphic software (Image Focus ${ }^{\circledR} 4.0$ ). On the other hand, length images of printed patterns with number of printing-over runs of $60,70,80,90$, and 100 were taken using a Scanning Electron Microscope (SEM) with 600x magnification, and their heights were measured using a graphic software (Motic $₫$ Images Plus 2.0). The number of printing-over runs that would give a width of 200 micrometer and its corresponding height and aspect ratio were calculated from the measurements. This would be the selected mold for the PDMS microchannel.

\section{Fabrication of PDMS Microchannel Using Soft Lithography}

The PDMS mixture was prepared with 10:1 ratio of the PDMS base and curing agent. The selected mold was placed on a polypropylene container and was wrapped with aluminum foil. The PDMS mixture was then poured on the container with the mold and degassed in a vacuum oven with a vacuum pressure of $0.5 \mathrm{~Pa}$ for 1 hour. Afterwards, the vacuum oven was pressurized to atmospheric pressure, and the PDMS was cured for 1.5 hours at $100^{\circ} \mathrm{C}$. The PDMS was cooled for 30 minutes and carefully peeled off from the container with the mold.

\section{E. PDMS Microchannel Characterization}

Cross sectional view images of PDMS microchannels were taken using SEM with 600x magnification, and their heights and widths were measured using a graphic software (Motic $®$ Images Plus 2.0).

\section{F. Construction of Microfluidic System}

The PDMS microchannel were cut to fit on a glass slide and punched using an improvised puncher. Silicon microtubings were connected to the holes of the PDMS microchannel using a cyanoacrylate adhesive (Pioneer® Mighty Bond® Instant Glue). These were then soaked in $100 \%$ dehydrated ethanol for 5 minutes and were further cleaned using a transparent tape. Afterwards, a PDMS microchannel was gently pressed manually on a glass slide.

\section{G. Fluid Flow Inspection}

The insulin syringe of the improvised microsyringe pump was filled with the prepared methylene blue solution. The PDMS microchannel was connected to the improvised microsyringe pump using a cyanoacrylate adhesive (Pioneer® Mighty Bond® Instant Glue). The sample fluid flow along the PDMS microchannel was viewed in a light microscope (Euromex Novex B Series) with 100x magnification.

\section{RESULTS AND DISCUSSION}

\section{A. Mold Characterization}

The mold produced from the printed relief features are characterized according to the width and aspect ratio. Measurements of the dimensions of the master mold done using the graphic softwares showed a linear correlation between the width, $w$, and the number of printing-over runs, $p$, as illustrated in Fig. 1.

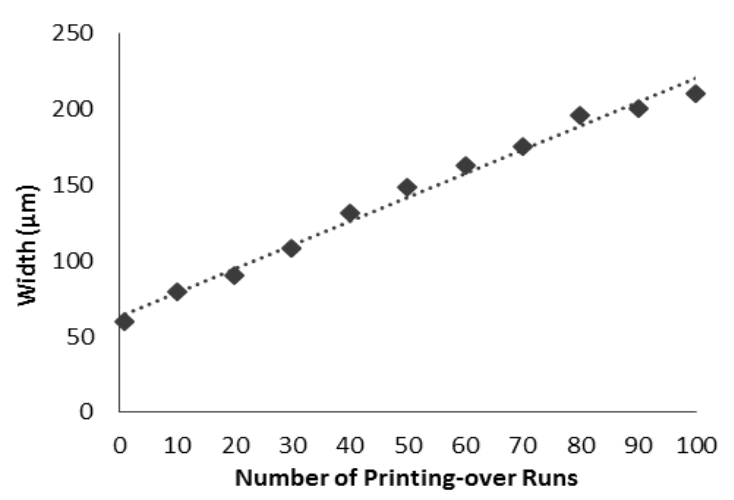

Fig. 1. Width as a function of number of printing-over runs.

The linear fit is given by the equation:

$$
w=1.5692 p+63.526
$$

with a correlation coefficient value $\left(\mathrm{r}^{2}\right)$ of 0.9888 . Based from (1), 86 printing-over runs was found to give a width of 200 micrometers, which is the defined maximum width for a microchannel based from reference [10].

It was observed in Fig. 2 that the aspect ratio of the mold increases as the number of printing-over runs is increased. The linear fit of the aspect ratio, $a$, as a function of the number of printing-over runs is given by the equation:

$$
a=0.00068 p+0.01628
$$

with a correlation coefficient $\left(\mathrm{r}^{2}\right)$ value of 0.96055 . With 86 
printing-over runs to be substituted in (2), the calculated aspect ratio is 0.07 .

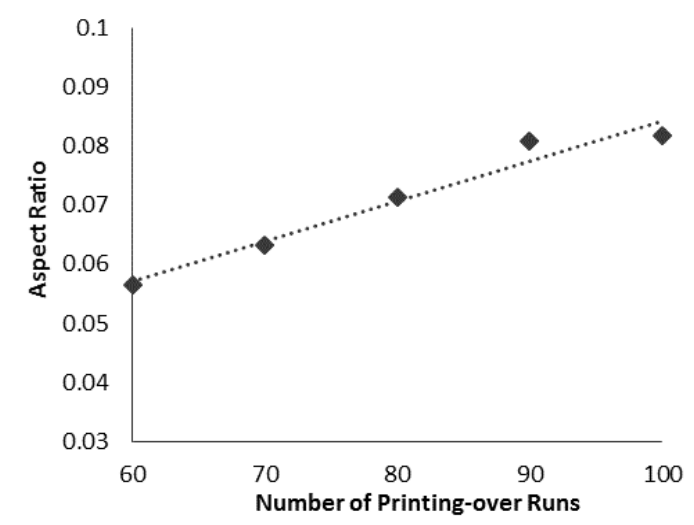

Fig. 2. Aspect ratio as a function of number of printing-over runs.

From microscope images of the mold shown in Fig. 3, irregularities on the width and height can be observed all throughout the length of the mold. This is due to the mechanism of the inkjet printer wherein small droplets of ink are propelled onto the paper through tiny nozzles. The irregularities could also be attributed to the pigment molecules of the ink which actually give the relief features of the mold.

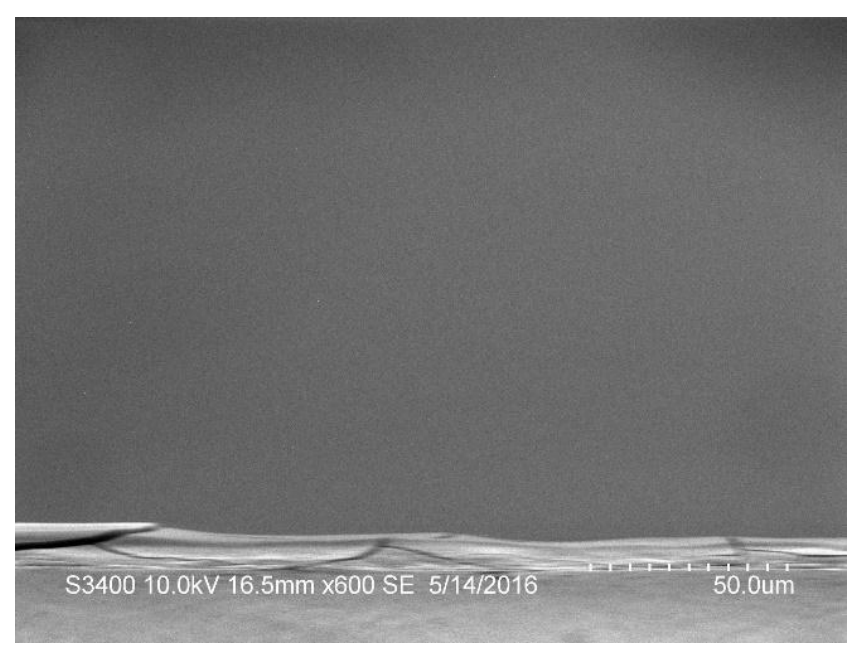

(a)

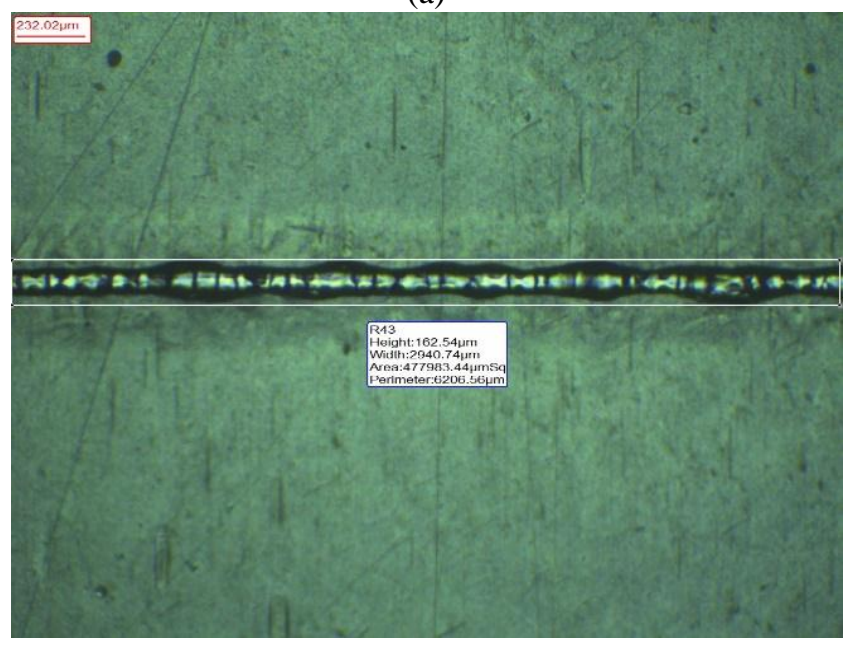

(b)

Fig. 3. Microscope images of the mold (60 printing-over runs) using (a) Scanning Electron Microscope (SEM), and (b) light microscope.

\section{B. PDMS Microchannel Characterization}

The mold produced from 86 printing-over runs is then used for soft lithography to fabricate a PDMS microchannel. The resulting PDMS microchannel has also undergone characterization by determining the width, height, and aspect ratio.

The produced PDMS microchannel has dimensions of 155 by 10 micrometers with aspect ratio equal to 0.06 . The cross-section of the microchannel is shown in Fig. 4.

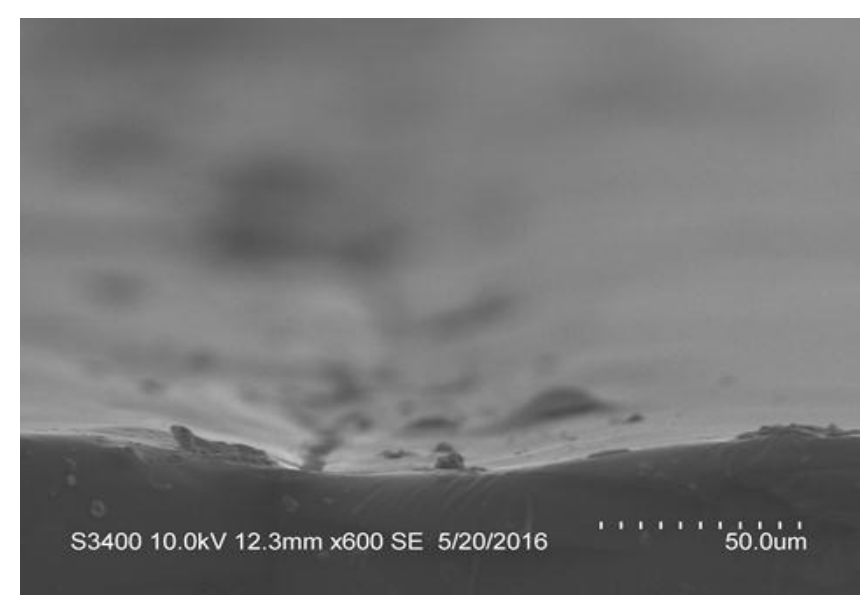

Fig. 4. SEM image of the PDMS microchannel.

In comparison with aspect ratios obtained from the previous studies that used inkjet printing: reference [8] with 0.008 and reference [9] with less than 0.008 - the improved inkjet printing process has increased the aspect ratio without having to modify the ink or the substrate. Also, fluid flow was successfully done which would have been impossible for a PDMS microchannel made by the unmodified inkjet printing (i.e. mold pattern printed once with normal inkjet ink).

\section{Cost Analysis}

A rough cost analysis was conducted based on the study in [11]. UV-LIGA SU-8 was chosen as representative of photolithography since it is one of the most common techniques used [11]. A unit produced from the improved PAP method is defined as one microchannel while a unit produced for UV LIGA SU-8 is one wafer [11]. The cost per unit of a fabrication method was computed using the given equation:

$$
C_{M}=\frac{C_{F}}{N}+C_{V}
$$

where $C_{M}$ is the cost per unit of a method, $C_{F}$ is the fixed cost, $N$ is the number of units, and $C_{V}$ is the variable cost per unit.

TABLE I: SUMMARY OF CAPITAL AND VARIABLE COSTS OF
\begin{tabular}{lcc} 
INKJET PRINTING AND UV LIGA SU-8 (IN PHILIPPINE PESO) \\
\hline \hline Cost & $\begin{array}{c}\text { Inkjet } \\
\text { Printing }\end{array}$ & $\begin{array}{c}\text { UV LIGA } \\
\text { SU-8 }\end{array}$ \\
\hline $\begin{array}{l}\text { Capital Cost } \\
\text { Variable Cost (per } \\
\text { channel/wafer) }\end{array}$ & 1,895 & $10,209,063$ \\
\hline \hline
\end{tabular}

From (3), considering the capital and variable costs of equipment and consumables as shown in Table I, the 
improved inkjet printing method is relatively much cheaper than the conventional method.

\section{CONCLUSIONS}

The selected mold is produced by 86 printing-over runs and generates a height of relief up to 14.8 micrometer for 200 micrometer width with an aspect ratio of 0.07 . The produced PDMS microchannel has dimensions of 155 by 10 micrometers with aspect ratio equal to 0.06 . Using the devised printing-over technique, fabrication of microchannel from an inkjet-ink-based mold has been enabled by the improvement of the aspect ratio. It is also determined that the aspect ratio increases proportionally along with increasing number of printing-over runs.

The capital and variable cost of the PAP inkjet method at 1,895 Philippine Peso (PHP) and 0.10 PHP per channel, respectively, are significantly lower than that of photolithography (UV LIGA-SU8) at 10.2 million PHP and 125.08 PHP per wafer, respectively. Along with a much lower cost of fabrication, the main materials used in the process are locally available and can be procured easily (e.g. office printer, sticker paper).

The fabricated microchannels, as seen from the characterization images, have limitations in the uniformity and smoothness of the surface. Thus, experimenting on different ink and paper combinations can be explored to minimize the observed roughness and irregularities. For further analysis of the microchannel, it is also suggested to conduct flow characterization.

The study was successful to the extent that the objectives were met by successful production of a microchannel for a much lower cost of fabrication and successfully inducing fluid flow across it. Further development should be focused on the fabrication of a functioning microfluidic device in order to prove the improved PAP method to be a viable low-cost alternative to the current fabrication standards.

\section{ACKNOWLEDGMENT}

We would like to extend our gratitude to the UP Engineering Research and Development Foundation, Inc. (ERDFI) through the Maynilad Professorial Chair Award (PCA) for the support they have provided for the completion of this study.

\section{REFERENCES}

[1] R. Ghodssi and P. Lin, MEMS Materials and Processes Handbook, New York: Springer Science, 2011.

[2] C. Hong, D. Bao, M. S. Thomas, J. M. Clift, and V. I. Vullev, "Print-and-peel fabrication of microelectrodes," Langmuir, vol. 24, pp. 8439-8442, July 2008.

[3] M. S. Thomas, B. Millare, J. M. Lift, D. Bao, C. Hong, and V. I. Vullev, "Print-and-peel fabrication for microfluidics: What's in it for biomedical applications?" Annals of Biomedical Engineering, vol. 38, no. 1, pp. 21-32, November 2009.

[4] N. Bao, Q. Zhang, J. J. Xu, and H. Y. Chen, "Fabrication of poly(dimethylsiloxane) microfluidic system based on masters directly printed with an office laser printer," Journal of Chromatography A, vol. 1089, pp. 270-275, October 2005.

[5] M. L. Branham, R. Tran-Son-Tay, C. Schoonover, P. S. Davis, S. D. Allen, and W. Shyy, "Rapid prototyping of micropatterned substrates using conventional laser printers," Journal of Materials Research, vol. 17 , pp. 1559-1562, July 2002.
[6] V. I. Vulley, J. Wan, V. Heinrich, P. Landsman, P. E. Bower, B. Xia, B. Millare, and G. Jones II, "Nonlithographic fabrication of microfluidic devices," Journal of the American Chemical Society, vol. 128, pp. 16062-16072, November 2006.

[7] G. V. Kaigala, S. Ho, R. Penterman, and C. J. Backhouse, "Rapid prototyping of microfluidic devices with a wax printer," Lab on a Chip, vol. 7, pp. 384-387, January 2007.

[8] C. Martin, A. Llobera, T. Leichle et al., "Novel methods to pattern polymers for microfluidics," Microelectronic Engineering, vol. 85, pp. 972-975, May 2008.

[9] Y. Xia and R. H. Friend, "Nonlithographic patterning through inkjet printing via holes," Applied Physics Letters, vol. 90, pp. 253513-1-253513-3, 2007

[10] S. G. Kandlikar, S. Garimella, D. Li, S. Colin, and M. R. King, Heat Transfer and Fluid Flow in Minichannels and Microchannels, Singapore: Elsevier Pte Ltd, 2006.

[11] R. A. Lawes, "Manufacturing costs for microsystems/MEMS using high aspect ratio microfabrication techniques," Microsystem Technologies, vol. 13, pp. 85-95, January 2007.



Kristian July R. Yap holds a M.S. in chemical engineering (2014) and a B.S. in chemical engineering (2009) from the University of the Philippines (UP), Diliman, Quezon City. He is currently an assistant professor of the Department of Chemical Engineering, teaching undergraduate chemical engineering courses, aside from being the head of UP Diliman's Task Force on Solid Waste Management. He is also affiliated with the Fuels, Energy, and Thermal Systems (FETS) laboratory of the department. Among his research interests are microfluidics, photocatalytic reaction engineering, and computational fluid dynamics.

Asst. Prof. Yap is a member of the Philippine Institute of Chemical Engineers (PIChE) and the Water Environment Association of the Philippines, Inc. He has been the holder of the Maynilad Professorial Chair Award for the past three years, and was given the Limcaoco Young Instructor Award for Teaching Excellence for the Academic Year 2011-2012 by the College of Engineering, University of the Philippines Diliman. 